\title{
Performance Analysis of a 3-2-1 Pose Estimation Device
}

\author{
Federico Thomas, Erika Ottaviano, Lluís Ros, and Marco Ceccarelli
}

\begin{abstract}
This paper deals with the problem of estimating the pose of a moving rigid body by measuring the length of six wires attached to it. Since wires can be seen as extensible legs, this problem is equivalent to that of solving the forward kinematics of a six-degree-of-freedom parallel manipulator. Among all possible locations for the attachments on the moving object, the "3-2-1" configuration is shown to exhibit a large number of favorable properties. The performance analysis of this particular configuration is addressed by finding analytic expressions for the estimated pose covariance matrix and the expected value of the pose estimation error, or bias error, which has been omitted in the previous analysis of wire-based tracking devices. This analysis takes advantage of a formulation for trilateration based on Cayley-Menger determinants, which is mathematically more tractable compared to previous ones, because all terms involved are determinants with geometric meaning. This accommodates a more thorough investigation of the properties of the device.
\end{abstract}

Index Terms-Bias error, distance geometry, kinematics singularities, parallel manipulators, pose measuring devices.

\section{INTRODUCTION}

$\mathbf{M}$ ANY systems for measuring the pose, i.e., position and orientation, of moving objects, also known as tracking systems, have been developed. They can be classified according to the measuring principle and used technology. Most of these systems are based on cameras, theodolites, lasers, and wires which are able to provide distance measurements by triangulation or trilateration techniques. Trilateration and triangulation determine the relative position between points by using the geometry of triangles or tetrahedra. Triangulation uses measurements of both distances and angles, whereas trilateration uses only distance measurements.

Tracking systems can also be classified according to their characteristics, such as accuracy, resolution, cost, measurement range, portability, and calibration requirements. Laser tracking systems exhibit good accuracy, which can be less than $1 \mu \mathrm{m}$ if the system is well calibrated. Unfortunately, this kind of system is very expensive, their calibration procedure is time-consuming, and they are sensitive to the environment.

Manuscript received March 26, 2004; revised July 14, 2004. This paper was recommended for publication by Associate Editor J. Angeles and Editor I. Walker upon evaluation of the reviewers' comments. This work was supported in part by the Italian-Spanish Bilateral CSIC-CNR Cooperation Program and the Spanish CICYT under Contract TIC2000-0696.

F. Thomas and L. Ros are with the Industrial Robotics Institute, Spanish High Council for Scientific Research (CSIC-UPC), 08028 Barcelona, Spain (e-mail: fthomas@iri.upc.edu; 1lros@iri.upc.edu).

E. Ottaviano and M. Ceccarelli are with the Laboratorio di Robotica e Meccatronica, Università degli Studi di Cassino, 03043 Cassino, Italy (e-mail: ottaviano@unicas.it; ceccarelli@unicas.it).

Digital Object Identifier 10.1109/TRO.2004.840894
Vision systems can reach an accuracy of $0.1 \mathrm{~mm}$. They are low-cost portable devices but their calibration procedure can be complicated. Wire-based systems can reach an accuracy of $0.1 \mathrm{~mm}$, and they are also low-cost portable devices but capable of measuring large displacements. Moreover, they exhibit a good compromise among accuracy, measurement range, cost, and operability.

Wire-based tracking devices consist of a fixed base and a platform connected by six wires whose tension is maintained, while the platform is moved, by pulleys and spiral springs on the base, where a set of encoders give the length of the wires. They can be modeled as six-degrees-of-freedom (6-DOF) parallel manipulators because wires can be seen as extensible legs connecting the platform and the base by means of spherical and universal joints, respectively.

Dimension deviations due to fabrication tolerances, wire-length uncertainties, or wire slackness may result in unacceptable performance of a wire-based tracking device. In general, the effects of all systematic errors can be eliminated by calibration. Some techniques for specific errors have already been proposed in the literature. For example, a method for compensating the cable guide outlet shape of wire encoders is detailed in [1], and a method for compensating the deflections caused by wire self-weights is described in [2]. In this paper, we will only consider wire-length errors that cannot be compensated because of their random nature.

Another indirect source of error is the force exerted by the measuring device itself. Indeed, all commercial wire encoders are designed to keep a large string tension. This is necessary to ensure that the inertia of the mechanism does not result in a wire going slack during a rapid motion. If a low wire force is used, it would reduce the maximum speed of the object to be tracked without the wires going slack. On the contrary, if a high wire force is used, the trajectory of the object to be tracked could be altered by the measuring device. Hence, a tradeoff between accuracy and speed arises.

An important issue in wire-based tracking devices is the number of attachments on the moving object and how many wires are connected to each attachment. On the base side, no two wire outlets can be made coincident because of physical limitations. This is not the case on the platform where it is advisable to reduce the number of attachments not only to derive simpler pose estimation expressions but also to reduce the risk of wire wrapping. If all attachments would collapse into a single point on the platform, wrapping problems can be avoided. Unfortunately, the minimum number of points for pose measurements is three. Moreover, the maximum number of wires attached to a point is also three, otherwise the lengths 


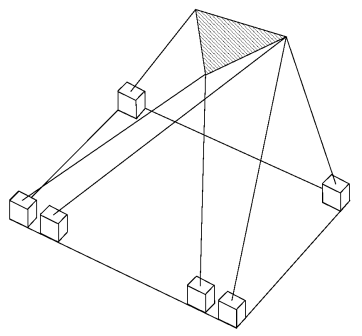

(a)

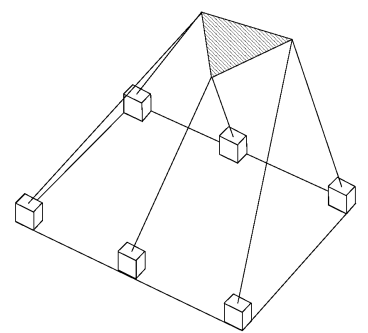

(b)
Fig. 1. (a) "3-2-1" and (b) "2-2-2" configurations for wire-based tracking devices.

of the wires will not be independent. This leads to only two possible configurations for the attachments on the moving object. Both have already been reported in the literature of wire-based tracking devices, namely, the following.

- The 2-2-2 configuration This configuration was first proposed in [2] for a wire-based tracking device [Fig. 1(b)]. The authors overlooked the fact that the kinematics of this configuration was already studied, for example, in [3], [4], and [5] where it was shown that its forward kinematics has 16 solutions. In other words, there are up to 16 poses for the moving object compatible with a given set of wire lengths. These configurations can only be obtained by a numerical method. The lack of a closed-form solution limits the analysis of the effects caused by the different forms of error.

- The 3-2-1 configuration This configuration was proposed in [1] [Fig. 1(a)]. The authors present it as a new configuration of a Stewart platform but the kinematics of this configuration was already studied, for example, in [6] and [7]. Its direct kinematics can be solved in closed-form by using three consecutive trilateration operations yielding eight solutions.

The two configurations above were compared, in terms of their sensitivity to wire-length errors, in [1]. The conclusion was that they have similar properties. Nevertheless, there are at least two reasons that make the 3-2-1 configuration preferable: 1 ) it has a lower number of forward kinematics solutions, which is useful to avoid ambiguities and 2) these solutions can be obtained by a closed-form formula, which enables us to perform an algebraic error analysis of the device.

As already mentioned, the forward kinematics solutions for the 3-2-1 configuration can be obtained by three consecutive trilateration operations. Although trilateration can be trivially expressed as the problem of finding the intersection of three spheres, different closed-form solutions have been proposed in the areas of computer graphics [8], robotics [9], aeronautics [10], computational geometry [11], and crystallography [12]. We rely on the formulation presented in [13] whose main advantage is that all terms involved are determinants with geometric interpretation, allowing a deeper insight into its error analysis.

This paper is organized as follows. Section II introduces the concept of Cayley-Menger bideterminants and gives a closed-form solution to the trilateration problem in terms of these determinants. Next, the forward kinematics and the singularities of the 3-2-1 configuration are analyzed in Section III.

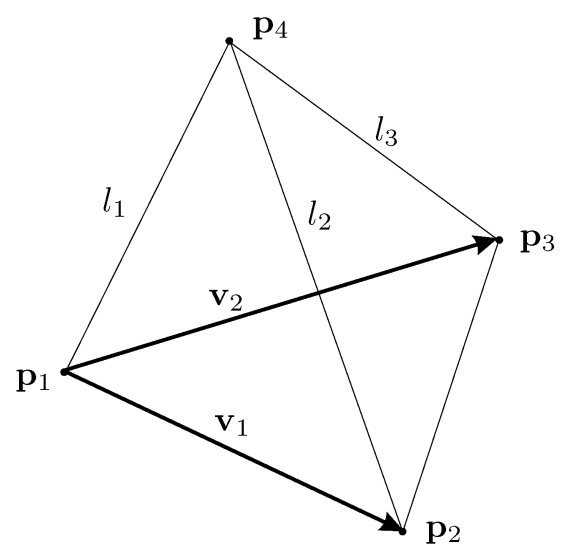

Fig. 2. Trilateration problem consists of given points $\mathbf{p}_{1}, \mathbf{p}_{2}$, and $\mathbf{p}_{3}$ and distances $l_{1}, l_{2}$, and $l_{3}$, locating $\mathbf{p}_{4}$.

The sensitivity of this configuration to wire-length errors and the effect of bias errors are tackled in Sections IV and V, respectively. Thanks to our formulation in terms of Cayley-Menger bideterminants, we will see how the bias error analysis has been greatly simplified. Experimental results are presented in Section VI that exemplify the most relevant characteristics of the 3-2-1 configuration analyzed throughout this paper. Finally, we conclude in Section VII, summarizing the main results and giving prospects for further research.

\section{CAYley-MEnger Bideterminants AND Trilateration}

The Cayley-Menger bideterminant of two sequences of $n$ points, $\left[\mathbf{p}_{1}, \ldots, \mathbf{p}_{n}\right]$ and $\left[\mathbf{q}_{1}, \ldots, \mathbf{q}_{n}\right]$, is defined as

$$
\begin{aligned}
& D\left(\mathbf{p}_{1}, \ldots, \mathbf{p}_{n} ; \mathbf{q}_{1}, \ldots, \mathbf{q}_{n}\right)= \\
& 2\left(\frac{-1}{2}\right)^{n} \cdot\left|\begin{array}{ccccc}
0 & 1 & 1 & 1 & 1 \\
1 & D\left(\mathbf{p}_{1}, \mathbf{q}_{1}\right) & D\left(\mathbf{p}_{1}, \mathbf{q}_{2}\right) & \cdots & D\left(\mathbf{p}_{1}, \mathbf{q}_{n}\right) \\
1 & D\left(\mathbf{p}_{2}, \mathbf{q}_{1}\right) & D\left(\mathbf{p}_{2}, \mathbf{q}_{2}\right) & \cdots & D\left(\mathbf{p}_{2}, \mathbf{q}_{n}\right) \\
\vdots & \vdots & \vdots & \ddots & \vdots \\
1 & D\left(\mathbf{p}_{n}, \mathbf{q}_{1}\right) & D\left(\mathbf{p}_{n}, \mathbf{q}_{2}\right) & \cdots & D\left(\mathbf{p}_{n}, \mathbf{q}_{n}\right)
\end{array}\right|
\end{aligned}
$$

where $D\left(\mathbf{p}_{i}, \mathbf{q}_{j}\right)$ denotes the squared distance between the points $\mathbf{p}_{i}$ and $\mathbf{q}_{j}$. This determinant plays a fundamental role in the so-called "Distance Geometry," a term coined by Blumenthal in [14] which refers to the analytical study of the Euclidean geometry in terms of invariants without resorting to artificial coordinate systems. Since in many cases of interest the two sequences of points are the same, it will be convenient to abbreviate $D\left(\mathbf{p}_{1}, \ldots, \mathbf{p}_{n} ; \mathbf{p}_{1}, \ldots, \mathbf{p}_{n}\right)$ by $D\left(\mathbf{p}_{1}, \ldots, \mathbf{p}_{n}\right)$, which is simply called a Cayley-Menger determinant. This determinant was first used by Cayley in 1841 [15], but it was not systematically studied until 1928, when Menger showed how it could be used to study convexity and other basic geometric properties [16].

Given three points in space, say $\mathbf{p}_{1}, \mathbf{p}_{2}$, and $\mathbf{p}_{3}$, the trilateration problem consists in finding the location of another point, say $\mathbf{p}_{4}$, whose distance to these three points is known (Fig. 2). According to the results presented in [13], $\mathbf{p}_{4}$ can be expressed as

$$
\mathbf{p}_{4}=\mathbf{p}_{1}+k_{1} \mathbf{v}_{1}+k_{2} \mathbf{v}_{2} \pm k_{3}\left(\mathbf{v}_{1} \times \mathbf{v}_{2}\right)
$$


where $\mathbf{v}_{1}=\mathbf{p}_{2}-\mathbf{p}_{1}, \mathbf{v}_{2}=\mathbf{p}_{3}-\mathbf{p}_{1}$, the \pm sign accounts for the two mirror symmetric solutions to the plane defined by $\mathbf{p}_{1}, \mathbf{p}_{2}$, and $\mathbf{p}_{3}$, and

$$
\begin{aligned}
& k_{1}=-\frac{D\left(\mathbf{p}_{1}, \mathbf{p}_{2}, \mathbf{p}_{3} ; \mathbf{p}_{1}, \mathbf{p}_{3}, \mathbf{p}_{4}\right)}{D\left(\mathbf{p}_{1}, \mathbf{p}_{2}, \mathbf{p}_{3}\right)} \\
& k_{2}=\frac{D\left(\mathbf{p}_{1}, \mathbf{p}_{2}, \mathbf{p}_{3} ; \mathbf{p}_{1}, \mathbf{p}_{2}, \mathbf{p}_{4}\right)}{D\left(\mathbf{p}_{1}, \mathbf{p}_{2}, \mathbf{p}_{3}\right)} \\
& k_{3}=\frac{\sqrt{D\left(\mathbf{p}_{1}, \mathbf{p}_{2}, \mathbf{p}_{3}, \mathbf{p}_{4}\right)}}{D\left(\mathbf{p}_{1}, \mathbf{p}_{2}, \mathbf{p}_{3}\right)}
\end{aligned}
$$

Many other formulations for trilateration are expressed according to a specific coordinate frame. For example, in [1], the $X Y$ plane is the plane defined by $\mathbf{p}_{1}, \mathbf{p}_{2}$, and $\mathbf{p}_{3}$, the $X$ axis is defined by the line containing $\mathbf{p}_{1}$ and $\mathbf{p}_{2}$ and the origin of the frame is located at $\mathbf{p}_{1}$. The formulation given here is coordinate-free. Nevertheless, the most interesting aspect of the proposed formulation compared to all others is that it is mathematically more tractable because all terms involved are determinants with geometric meaning. In fact,

$$
D\left(\mathbf{p}_{1}, \mathbf{p}_{2}, \mathbf{p}_{3}, \mathbf{p}_{4}\right)=36 V^{2}
$$

where $V$ is the volume of the tetrahedron defined by $\mathbf{p}_{1}$, $\mathbf{p}_{2}, \mathbf{p}_{3}$, and $\mathbf{p}_{4}$. Hence, $D\left(\mathbf{p}_{1}, \mathbf{p}_{2}, \mathbf{p}_{3}, \mathbf{p}_{4}\right)=0$ if, and only if, $\mathbf{p}_{1}, \mathbf{p}_{2}, \mathbf{p}_{3}$, and $\mathbf{p}_{4}$ lie on a plane. In addition,

$$
D\left(\mathbf{p}_{1}, \mathbf{p}_{2}, \mathbf{p}_{3}\right)=4 A^{2}
$$

where $A$ is the area of the triangle defined by points $\mathbf{p}_{1}, \mathbf{p}_{2}$, and $\mathbf{p}_{3}$. Hence, $D\left(\mathbf{p}_{1}, \mathbf{p}_{2}, \mathbf{p}_{3}\right)=0$ if, and only if, $\mathbf{p}_{1}, \mathbf{p}_{2}$, and $\mathbf{p}_{3}$, are aligned. Furthermore,

$$
D\left(\mathbf{p}_{1}, \mathbf{p}_{2}, \mathbf{p}_{3} ; \mathbf{q}_{1}, \mathbf{q}_{2}, \mathbf{q}_{3}\right)=2 A_{1} \cdot 2 A_{2} \cdot \cos \phi
$$

where $A_{1}$ and $A_{2}$ are the oriented areas of the triangles $\mathbf{p}_{1} \mathbf{p}_{2} \mathbf{p}_{3}$ and $\mathbf{q}_{1} \mathbf{q}_{2} \mathbf{q}_{3}$, respectively, and $\phi$ is the dihedral angle between the planes they define.

As a consequence of this geometric interpretation of all terms involved in the formulation, we will be able to accommodate a more thorough investigation of the effects caused by wire-length errors, singularities, and bias errors of a 3-2-1 configuration, as shown below.

\section{FORWARD KINEMATICS AND SINGULARITIES}

The direct kinematics of the 3-2-1 configuration can be solved by three consecutive trilateration operations. Indeed, according to Fig. 3(a), given the wire lengths $l_{1}, l_{2}$, and $l_{3}$, there are two possible mirror locations for $\mathbf{b}_{1}$ with respect to the plane defined by points $\mathbf{a}_{1}, \mathbf{a}_{2}$, and $\mathbf{a}_{3}$ [Fig. 3(b)]. Once one of these two solutions for $\mathbf{b}_{1}$ is chosen, $\mathbf{a}_{4}, \mathbf{a}_{5}, \mathbf{b}_{1}$, and $\mathbf{b}_{2}$ define another tetrahedron with known edge lengths. Again, there are two possible mirror locations for $\mathbf{b}_{2}$, in this case with respect to the plane defined by $\mathbf{a}_{4}, \mathbf{a}_{5}$, and $\mathbf{b}_{1}$ [Fig. 3(c)]. Finally, after choosing one of the two solutions, $\mathbf{a}_{6}, \mathbf{b}_{1}, \mathbf{b}_{2}$, and $\mathbf{b}_{3}$ define another tetrahedron with known edge lengths. In this case there are two possible mirror locations for $\mathbf{b}_{3}$ with respect to the plane defined by $\mathbf{a}_{6}, \mathbf{b}_{1}$, and $\mathbf{b}_{2}$ [Fig. 3(d)].

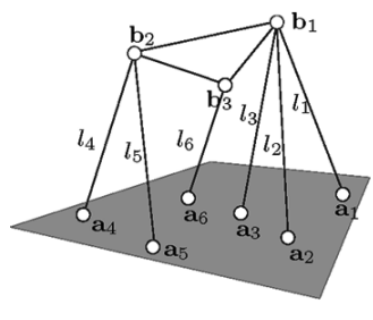

(a)

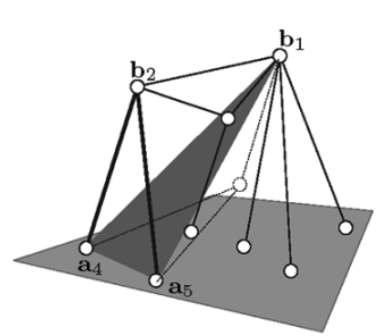

(c)
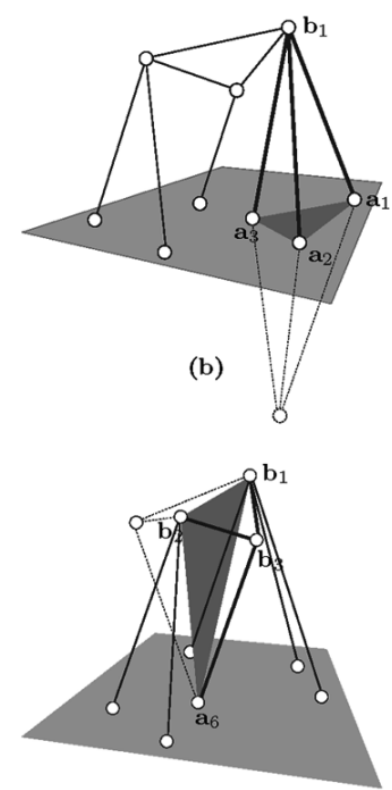

(d)
Fig. 3. (a) General kinematic model of a 3-2-1 tracking system. There are up to eight possible solutions for the configuration of the platform compatible with a set of wire lengths due to the two possible solutions for the location of (b) $\mathbf{b}_{1}$, (c) $\mathbf{b}_{2}$, and (d) $\mathbf{b}_{3}$.

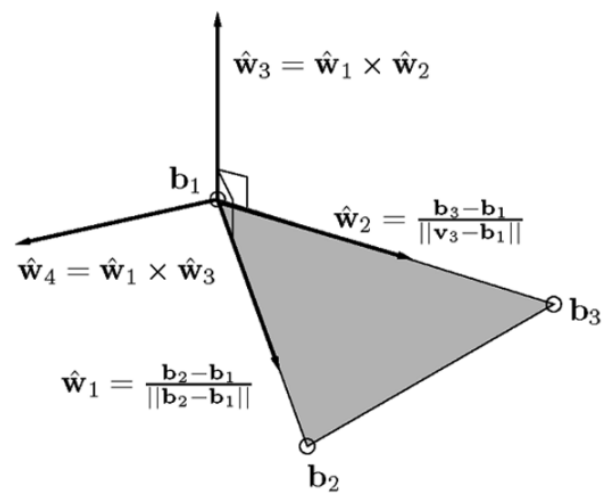

Fig. 4. Reference frame $\left(\hat{\mathbf{w}}_{1}, \hat{\mathbf{w}}_{3}, \hat{\mathbf{w}}_{4}\right)$ associated with the moving platform.

Once points $\mathbf{b}_{1}, \mathbf{b}_{2}$, and $\mathbf{b}_{3}$ have been located, they can be used to define a reference frame on the moving object. For example, according to Fig. 4, the $x$ axis can be defined by the direction given by $\hat{\mathbf{w}}_{1}$, the $y$ axis can be chosen orthogonal to the plane defined by $\hat{\mathbf{w}}_{1}$ and $\hat{\mathbf{w}}_{2}$, and the $z$ axis can be obtained to give a Cartesian reference frame. These vectors form a set of orthogonal basis vectors whose directions are known relative to both the fixed and the moving reference frames. Note that this completely solves the forward kinematics of the 3-2-1 configuration without evaluating any trigonometric functions.

There are certain singular sets of wire lengths in which the number of solutions, for at least one of the three trilateration operations, is not two. For example, according to (1) and (2), the first trilateration will yield only one solution if, and only if, $D\left(\mathbf{a}_{1}, \mathbf{a}_{2}, \mathbf{a}_{3}, \mathbf{b}_{1}\right)=0$, i.e., if $\mathbf{a}_{1}, \mathbf{a}_{2}, \mathbf{a}_{3}$, and $\mathbf{b}_{1}$ lie on the same plane. Also, the result is undefined if, and only if, $D\left(\mathbf{a}_{1}, \mathbf{a}_{2}, \mathbf{a}_{3}\right)=0$, i.e., if $\mathbf{a}_{1}, \mathbf{a}_{2}$, and $\mathbf{a}_{3}$ are aligned. Since $D\left(\mathbf{a}_{1}, \mathbf{a}_{2}, \mathbf{a}_{3}\right)=0$ implies $D\left(\mathbf{a}_{1}, \mathbf{a}_{2}, \mathbf{a}_{3}, \mathbf{b}_{1}\right)=0$, this latter condition encompasses all singularities for the first trilateration. 
This reasoning can be repeated for the other two trilaterations concluding that, if the platform is in a configuration in which at least one of the following three equations is satisfied:

$$
\begin{aligned}
& D\left(\mathbf{a}_{1}, \mathbf{a}_{2}, \mathbf{a}_{3}, \mathbf{b}_{1}\right)=0 \\
& D\left(\mathbf{a}_{4}, \mathbf{a}_{5}, \mathbf{b}_{1}, \mathbf{b}_{2}\right)=0
\end{aligned}
$$

or

$$
D\left(\mathbf{a}_{6}, \mathbf{b}_{1}, \mathbf{b}_{2}, \mathbf{b}_{3}\right)=0
$$

then the tracking system is in a singularity. In other words, these three determinants fully characterize all of the singularities and, hence, a compact condition to test whether the system is in a singularity or not is given by

$$
D\left(\mathbf{a}_{1}, \mathbf{a}_{2}, \mathbf{a}_{3}, \mathbf{b}_{1}\right) D\left(\mathbf{a}_{4}, \mathbf{a}_{5}, \mathbf{b}_{1}, \mathbf{b}_{2}\right) D\left(\mathbf{a}_{6}, \mathbf{b}_{1}, \mathbf{b}_{2}, \mathbf{b}_{3}\right)=0
$$

which will be useful later.

Each equation in (6) implicitly defines an algebraic variety of dimension 5 that divides the configuration space of the platform, $\mathbb{R}^{3} \times \mathrm{SO}(3)$, into two half-spaces. Then, the three equations in (6) lead to a partition of this configuration space into regions. During normal operation, the tracking system should work in one of these regions without getting out of it to avoid ambiguities. Unfortunately, this is not the only reason to avoid a singularity. Near a singularity, small errors in the wire lengths induce important errors in the pose estimations not only in terms of their variances (this is discussed in Section IV) and also in their biases (this is discussed in Section V).

\section{SENSITIVITY TO WIRE-LENGTH ERRORS}

In what follows, we will assume that the measured wire lengths are corrupted by random noise with Gaussian probability density function and we will show how this error contributes to the measured pose error.

Let us suppose that the platform is in a given pose, say $\mathbf{q}^{0} \in$ $\mathbb{R}^{3} \times \mathrm{SO}(3)$, and the corresponding wire lengths for this pose are $\mathbf{1}^{0}=\left(\begin{array}{llll}l_{1}^{0} & l_{2}^{0} & \cdots & l_{6}^{0}\end{array}\right)^{T}$. In practice, we are unable to measure these wire lengths. Instead, we get values contaminated by additive random noise, which can be expressed in vector form as $\mathbf{l}=\mathbf{l}^{0}+\delta \mathbf{l}$, where $\delta \mathbf{l}=\left(\begin{array}{llll}\delta l_{1}^{0} & \delta l_{2}^{0} & \cdots & \delta l_{6}^{0}\end{array}\right)^{T}$ is the vector of additive random errors.

The wire-length errors are assumed to have zero mean value, that is

$$
E\{\delta 1\}=0
$$

where $E\{\cdot\}$ stands for the expected value operation. We also assume that the wire-length errors are uncorrelated with the same variance $\sigma_{r}^{2}$. Consequently their covariance matrix can be expressed as

$$
E\left\{\delta \mathbf{l} \delta \mathbf{1}^{T}\right\}=\sigma_{r}^{2} \mathbf{I}
$$

where $\mathbf{I}$ denotes the identity matrix.

Now, the problem is to compute the error in the position and

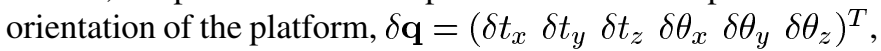

induced by the errors in the wire lengths $\delta 1$. For small wirelength errors, if the reference frame is taken so that $\mathbf{q}^{0}=\mathbf{0}$, it can be proven that

$$
\delta \mathbf{1}=\mathbf{J} \delta \mathbf{q}
$$

where the rows of $\mathbf{J}$ are the Plücker coordinates of the wire lines [17]. Hence

$$
E\left\{\delta \mathbf{l} \delta \mathbf{1}^{T}\right\}=\mathbf{J} E\left\{\delta \mathbf{q} \delta \mathbf{q}^{T}\right\} \mathbf{J}^{T}=\sigma_{r}^{2} \mathbf{I} .
$$

Finally, the covariance matrix for the pose is

$$
E\left\{\delta \mathbf{q} \delta \mathbf{q}^{T}\right\}=\sigma_{r}^{2} \mathbf{J}^{-1}\left(\mathbf{J}^{T}\right)^{-1}=\sigma_{r}^{2}\left(\mathbf{J}^{T} \mathbf{J}\right)^{-1} .
$$

Note that (12) is well defined provided that $\mathbf{J}$ is nonsingular. Since the rows of $\mathbf{J}$ are Plücker coordinates of lines, it is possible to characterize the singularities of $\mathbf{J}$, i.e., configurations in which $\operatorname{det}(\mathbf{J})=0$, in terms of the geometry of linearly dependent sets of lines. The set of conditions is typical for many parallel manipulators [18]. Nevertheless, in our case, according to the results presented in [19] for the 3-2-1 configuration, we know that

$$
\operatorname{det}(\mathbf{J})=216 \frac{V_{1} V_{2} V_{3}}{l_{1} l_{2} l_{3} l_{4} l_{5} l_{6}}
$$

where $V_{1}, V_{2}$, and $V_{3}$ are the volumes of the tetrahedra $\mathbf{a}_{1} \mathbf{a}_{2} \mathbf{a}_{3} \mathbf{b}_{1}, \mathbf{a}_{4} \mathbf{a}_{5} \mathbf{b}_{2} \mathbf{b}_{3}$, and $\mathbf{a}_{6} \mathbf{b}_{1} \mathbf{b}_{2} \mathbf{b}_{3}$, respectively. Then, using (3)

$\operatorname{det}(\mathbf{J})=$

$$
\frac{\sqrt{D\left(\mathbf{a}_{1}, \mathbf{a}_{2}, \mathbf{a}_{3}, \mathbf{b}_{1}\right) D\left(\mathbf{a}_{4}, \mathbf{a}_{5}, \mathbf{b}_{1}, \mathbf{b}_{2}\right) D\left(\mathbf{a}_{6}, \mathbf{b}_{1}, \mathbf{b}_{2}, \mathbf{b}_{3}\right)}}{l_{1} l_{2} l_{3} l_{4} l_{5} l_{6}}
$$

is true, which fully agrees with the characterization of the singularities given by (7), obtained using pure geometric arguments.

The platform pose error is contained in the ellipsoid

$$
\left\{\delta \mathbf{q} \in \mathbb{R}^{3} \times \mathrm{SO}(3) \mid \delta \mathbf{q}^{T} \mathbf{J}^{T} \mathbf{J} \delta \mathbf{q} \leq 16.8 \sigma_{r}^{2}\right\}
$$

with probability 0.99 [20, p. 697]. Then, although $\mathbf{J}^{T} \mathbf{J}$ does not make physical sense because its elements are obtained by adding length-squared and dimensionless quantities, it still seems reasonable to use its volume [21, pp. 103-104], that is

$$
\frac{\pi^{3}}{6 \sqrt{\operatorname{det}\left(\mathbf{J}^{T} \mathbf{J} / 16.8 \sigma_{r}^{2}\right)}} \simeq \frac{2370.8 \sigma_{r}^{6}}{\operatorname{det}(\mathbf{J})}
$$

as the measurement of the sensitivity of the pose error with respect to wire-length errors. Then, using (14) and (16), and removing all constant factors, we define

$$
\mathcal{S}=\frac{l_{1} l_{2} l_{3} l_{4} l_{5} l_{6}}{\sqrt{D\left(\mathbf{a}_{1}, \mathbf{a}_{2}, \mathbf{a}_{3}, \mathbf{b}_{1}\right) D\left(\mathbf{a}_{4}, \mathbf{a}_{5}, \mathbf{b}_{1}, \mathbf{b}_{2}\right) D\left(\mathbf{a}_{6}, \mathbf{b}_{1}, \mathbf{b}_{2}, \mathbf{b}_{3}\right)}}
$$

as a sensitivity index of the 3-2-1 configuration to wire-length errors. Note that this index is independent from the chosen reference frames and, what is more important, it can be interpreted 
either as the volume of the uncertainty ellipsoid associated with the pose error, or as the reciprocal of the product of three tetrahedra volumes in the working space.

\section{BIAS ERROR}

Although the noise in the length measurements is assumed to have zero mean value, we show in this section that the expected value of the estimation error obtained by trilateration does not equal zero due to nonlinearities. Moreover, this kind of error-technically known as bias error-is only attributable to wire-length errors.

We only analyze the bias error for a single trilateration keeping in mind that, in a 3-2-1 configuration, its effect is carried forward along the three consecutive trilaterations.

Following the analysis for the single trilateration operation shown in Fig. 2, the error in the location of $\mathbf{p}_{4}$, due to small wire-length errors, can be well approximated by retaining the terms up to the second-order partial derivatives in the Taylor expansion of (1), that is

$$
\delta \mathbf{p}_{4}=\mathbf{p}_{4}-\mathbf{p}_{4}^{0}=\sum_{i=1}^{3} \frac{\partial \mathbf{p}_{4}}{\partial l_{i}} \delta l_{i}+\frac{1}{2} \sum_{i=1}^{3} \sum_{j=1}^{3} \frac{\partial^{2} \mathbf{p}_{4}}{\partial l_{i} \partial l_{j}} \delta l_{i} \delta l_{j}
$$

The expected value of the random error in $\mathbf{p}_{4}$, i.e., the bias error, is

$$
E\left\{\delta \mathbf{p}_{4}\right\}=\frac{1}{2} \sum_{i=1}^{3} \sum_{j=1}^{3} \frac{\partial^{2} \mathbf{p}_{4}}{\partial l_{i} \partial l_{j}} E\left\{\delta l_{i} \delta l_{j}\right\}
$$

Then, using (9)

$$
E\left\{\delta \mathbf{p}_{4}\right\}=\frac{\sigma_{r}^{2}}{2}\left(\frac{\partial^{2} \mathbf{p}_{4}}{\partial l_{1}^{2}}+\frac{\partial^{2} \mathbf{p}_{4}}{\partial l_{2}^{2}}+\frac{\partial^{2} \mathbf{p}_{4}}{\partial l_{3}^{2}}\right) .
$$

Finally, substituting (1)

$$
E\left\{\delta \mathbf{p}_{4}\right\}=\frac{\sigma_{r}^{2}}{2}\left(\nabla^{2} k_{1} \mathbf{v}_{1}+\nabla^{2} k_{2} \mathbf{v}_{2} \pm \nabla^{2} k_{3}\left(\mathbf{v}_{1} \times \mathbf{v}_{2}\right)\right)
$$

where $\nabla^{2} k_{i}=\partial^{2} k_{i} / \partial l_{1}^{2}+\partial^{2} k_{i} / \partial l_{2}^{2}+\partial^{2} k_{i} / \partial l_{3}^{2}$ and the + or sign is used depending on the chosen trilateration solution. The analytic expression for these derivatives can be found in [26]. Substituting them into (21), we obtain

$$
\begin{aligned}
E\left\{\delta \mathbf{p}_{4}\right\} & \\
= & \frac{\sigma_{r}^{2}}{2}\left[\frac{b^{2}}{8 A_{b}^{2}} \mathbf{v}_{1}+\frac{a^{2}}{8 A_{b}^{2}} \mathbf{v}_{2}\right. \\
& +\left(\frac{1}{6 V}+\frac{l_{1}^{2} c^{2}+l_{2}^{2} b^{2}+l_{3}^{2} a^{2}}{24 A_{b}^{2} V}-\frac{l_{1}^{2} A_{3}^{2}+l_{2}^{2} A_{2}^{2}+l_{3}^{2} A_{1}^{2}}{54 V^{3}}\right) \\
& \left.\times\left(\mathbf{v}_{1} \times \mathbf{v}_{2}\right)\right] .
\end{aligned}
$$

where $a$ and $b$ are the lengths of the edges $\mathbf{p}_{1} \mathbf{p}_{2}$ and $\mathbf{p}_{1} \mathbf{p}_{3}$, respectively, $A_{b}, A_{1}, A_{2}$, and $A_{3}$ are the areas of the triangles $\mathbf{p}_{1} \mathbf{p}_{2} \mathbf{p}_{3}, \mathbf{p}_{r} \mathbf{p}_{2} \mathbf{p}_{3}, \mathbf{p}_{r} \mathbf{p}_{1} \mathbf{p}_{3}$, and $\mathbf{p}_{r} \mathbf{p}_{1} \mathbf{p}_{2}$, respectively, where $\mathbf{p}_{r}$ is the orthogonal projection of $\mathbf{p}_{4}$ onto the plane defined

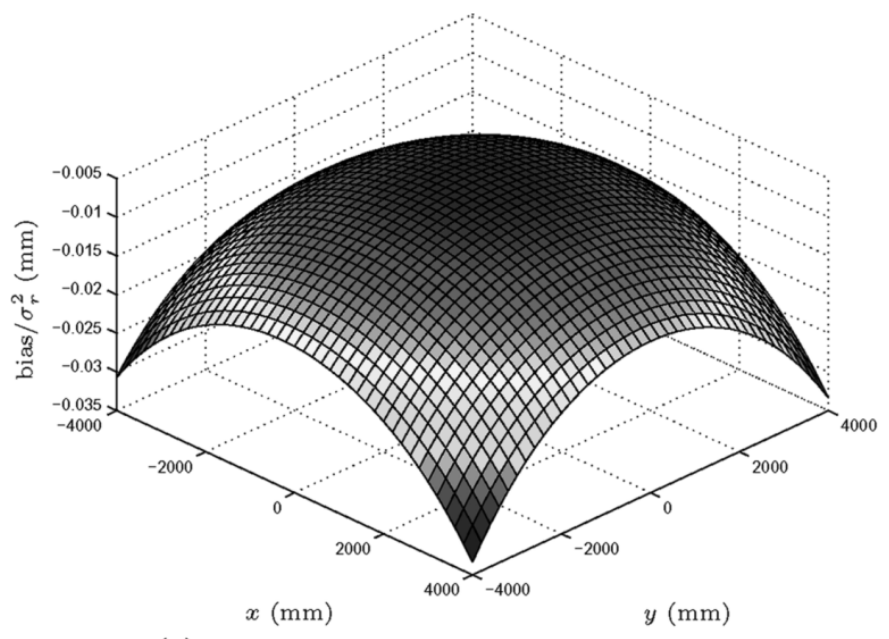

(a)

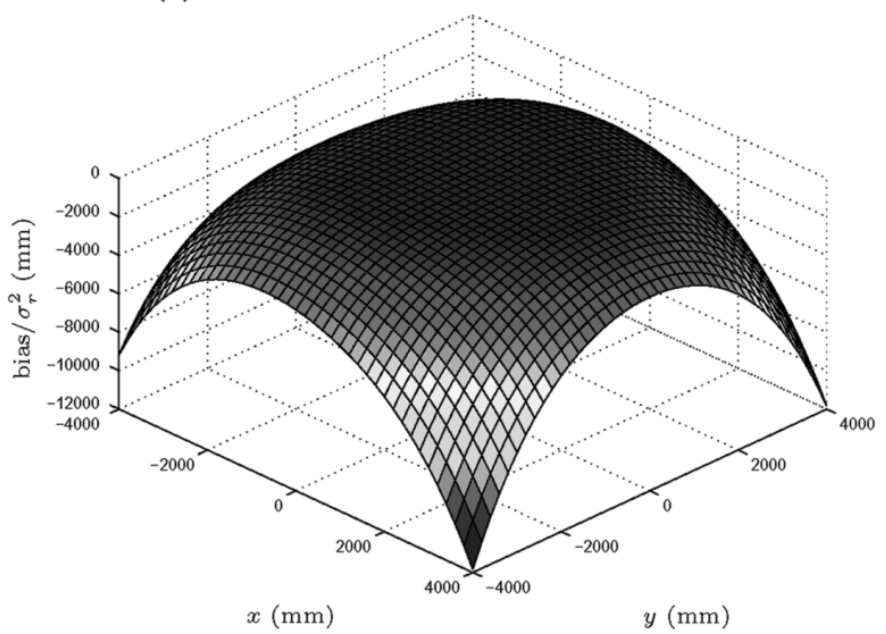

(b)

Fig. 5. For a representative example, component of the trilateration bias error orthogonal to the base plane for a distance of (a) 4000 and (b) $40 \mathrm{~mm}$ of the moving point with respect to the base plane (see the text for details).

by $\mathbf{p}_{1}, \mathbf{p}_{2}$, and $\mathbf{p}_{3}$, and $V$ is the volume of the tetrahedron $\mathbf{p}_{1} \mathbf{p}_{2} \mathbf{p}_{3} \mathbf{p}_{4}$.

It is important to realize that the terms multiplying $\mathbf{v}_{1}$ and $\mathbf{v}_{2}$ are constant, i.e., the bias error parallel to the base plane is constant, independently of the location of $\mathbf{p}_{4}$.

Let us examine this error for a representative case. Consider that the three base points form an equilateral triangle on the $\mathrm{XY}$ plane inscribed in a circle centered in the origin of radius $1000 \mathrm{~mm}$. The base points are $\mathbf{p}_{1}=(500 \sqrt{3},-500,0)^{T}, \mathbf{p}_{2}=$ $(0,1000,0)^{T}$, and $\mathbf{p}_{3}=(-500 \sqrt{3},-500,0)^{T}$. For this case, $\nabla^{2} k_{1}=\nabla^{2} k_{2}=1.35 \cdot 10^{-19} \mathrm{~mm}^{-2}$. Hence, the bias error parallel to the base plane can be neglected. In order to analyze the orthogonal component of this error to the base plane, let us assume that $\mathbf{p}_{4}$ sweeps an XY plane square area, a distance of $4000 \mathrm{~mm}$ from the base plane, spanning in each direction from -4000 to $4000 \mathrm{~mm}$. The maximum bias error in this region is $-0.03 \sigma_{r}^{2} \mathrm{~mm}$ [Fig. 5(a)], which can also be neglected in practice. This amount exponentially increases as $\mathbf{p}_{4}$ reduces its distance to the base plane, i.e., the singularity is approached. When the distance is $400 \mathrm{~mm}$, the maximum bias error is $-12 \sigma_{r}^{2}$, and 


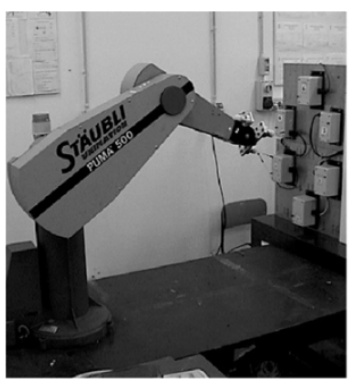

(a)

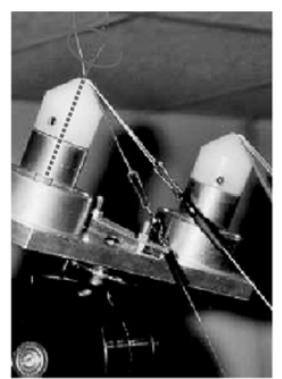

(b)

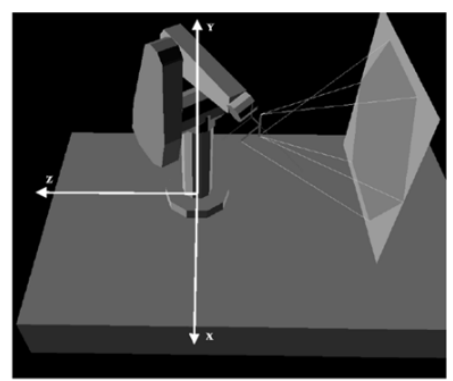

(c)

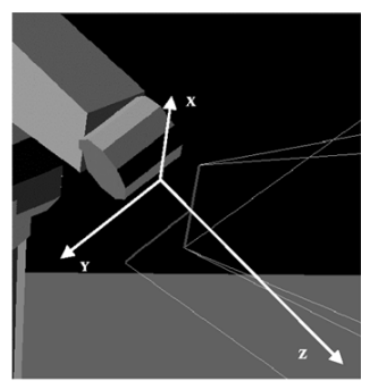

(d)

Fig. 6. (a) Experimental setup. (b) Detail of one of the attachments on the moving platform. (c)-(d) Graphics simulation of the tracking device connected to a PUMA robot.

when it is reduced to $40 \mathrm{~mm}$, the maximum bias error amounts to $-11842 \sigma_{r}^{2}$ [Fig. 5(b)].

In general, it can be proven that, as a consequence of this error, when $\mathbf{p}_{4}$ moves on a plane parallel to that of the three base points, the estimation will erroneously indicate that it ascends and descends when it approaches, and goes away from the barycenter of the base points, respectively. Note that, since this is a systematic error, it can be compensated by proper calibration.

The trilateration bias error was already examined in [10], in the context of aeronautic navigation, where two main results were drawn.

R1) The projection of the bias error onto the base plane can be neglected provided that $\mathbf{p}_{1}, \mathbf{p}_{2}$, and $\mathbf{p}_{3}$ form a nondegenerate triangle.

R2) The bias error becomes relevant as $\mathbf{p}_{4}$ approaches the base plane.

These results fully agree with the results obtained for the representative example analyzed above.

Contrary to all other formulations, these two facts have a direct accommodation in ours. Indeed, as a consequence of R1), (21) can be approximated by

$$
E\left\{\delta \mathbf{p}_{4}\right\} \simeq \pm \frac{\sigma_{r}^{2}}{2} \nabla^{2} k_{3}\left(\mathbf{v}_{1} \times \mathbf{v}_{2}\right)
$$

and, as a consequence of R2), this equation can be further simplified [26], near a singularity, into

$$
E\left\{\delta \mathbf{p}_{4}\right\} \simeq \mp \sigma_{r}^{2} \frac{l_{1}^{2} A_{3}^{2}+l_{2}^{2} A_{2}^{2}+l_{3}^{2} A_{1}^{2}}{54 V^{3}}\left(\mathbf{v}_{1} \times \mathbf{v}_{2}\right)
$$

This equation is remarkable because of its simplicity (compare it to the result presented in [10]).

We conclude that near a singularity not only are random errors amplified but also estimations are highly biased. As exemplified in the next section, the destructive combination of both effects makes any practical measurement near a singularity almost useless.

\section{EXPERIMENTAL RESULTS}

The validation of the theoretical results obtained has been carried out by tracking the motion of the end effector of a PUMA 562 robot, both using a real system [22] and a simulation tool [23] (Fig. 6). The simulations have proven to be of great in-
TABLE I

CoOrdinates (IN MiLlimeters) OF THE BASE AND PlatForm ARTICULATION CENTERS REFERRED TO THE FRAMES SHOWN IN FIG. 6(c) AND (d), RESPECTIVELY

\begin{tabular}{c||c}
\hline $\mathbf{a}_{1}$ & $(500,500,-1000)$ \\
\hline $\mathbf{a}_{2}$ & $(250,933,-1000)$ \\
\hline $\mathbf{a}_{3}$ & $(-250,933,-1000)$ \\
\hline $\mathbf{a}_{4}$ & $(-500,500,-1000)$ \\
\hline $\mathbf{a}_{5}$ & $(-250,67,-1000)$ \\
\hline $\mathbf{a}_{6}$ & $(250,67,-1000)$ \\
\hline
\end{tabular}

\begin{tabular}{c||c}
\hline $\mathbf{b}_{1}$ & $(-100,0,50)$ \\
\hline $\mathbf{b}_{2}$ & $(100,0,50)$ \\
\hline $\mathbf{b}_{3}$ & $(0,100,50)$ \\
\hline
\end{tabular}

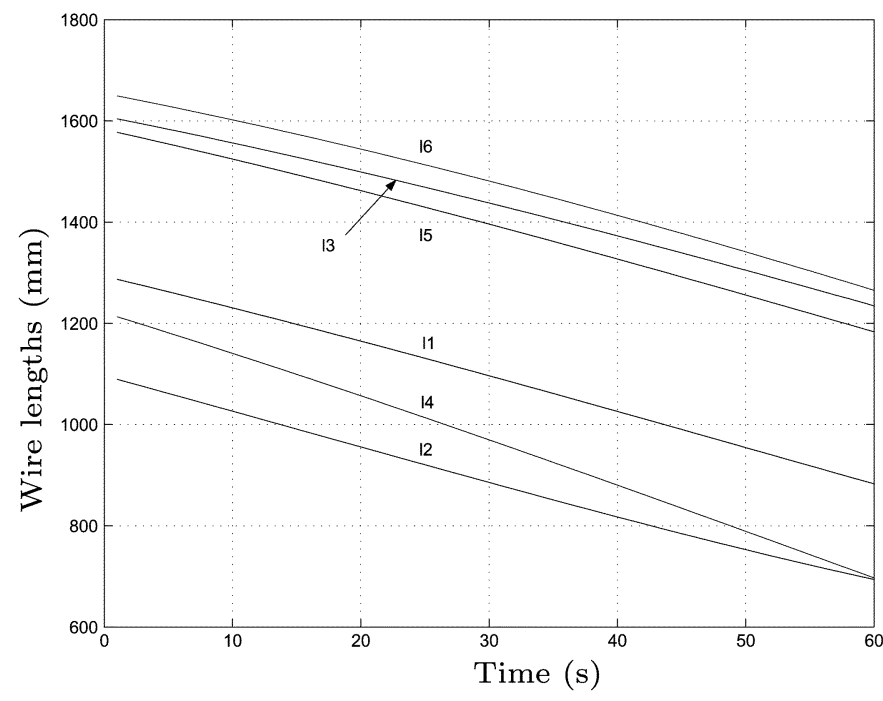

Fig. 7. First experiment. Evolution of the six wire lengths along the trajectory.

terest to compare the estimations obtained from noisy experimental data to those obtained without noise near singularities in simulation.

The implemented mechatronic system was designed at the Laboratory of Robotics and Mechatronics in Cassino where a prototype is currently in use for robot workspace evaluation [24]. It consists of a mechanical part and an interface connected to a personal computer. The signals from the wire transducers, which have a working range of $1.5 \mathrm{~m}$, are fed through an amplifier to a 12-b A/D board installed in the computer. The articulation centers on the base have been arranged forming a hexagon. Their coordinates, as well as those of the platform attachments, can be found in Table I. These coordinates are referenced to the frames shown in Fig. 6(b) and (c), respectively.

Due to physical limitations, the implemented wire-based device only works in one of the two half-spaces defined by the base 


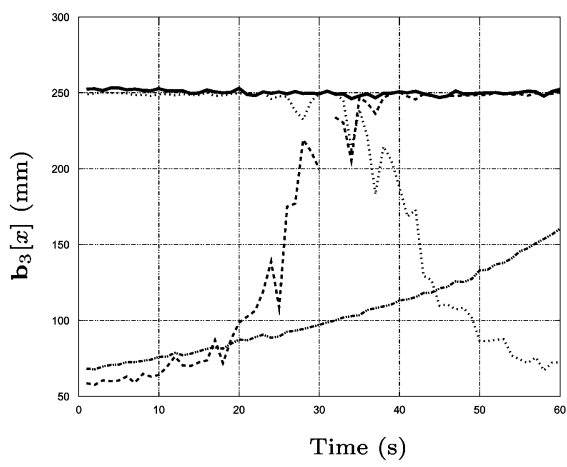

(a)

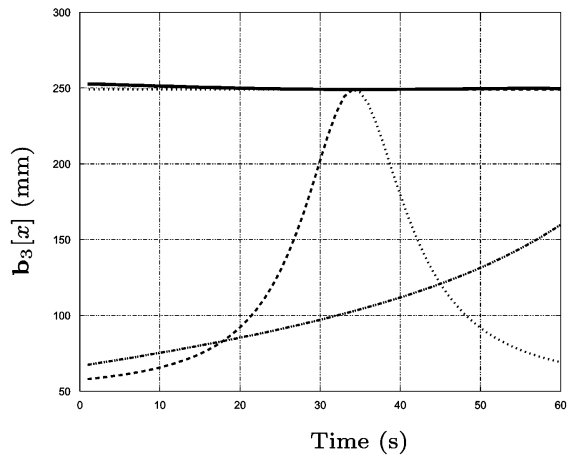

(d)

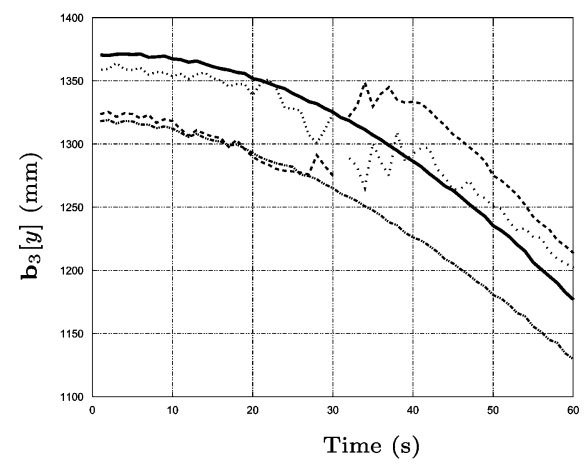

(b)

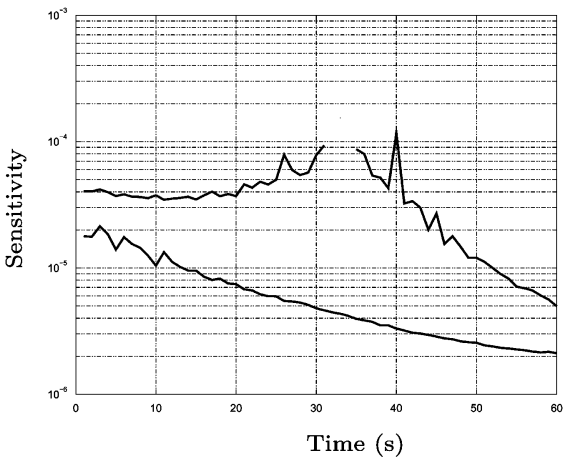

(e)

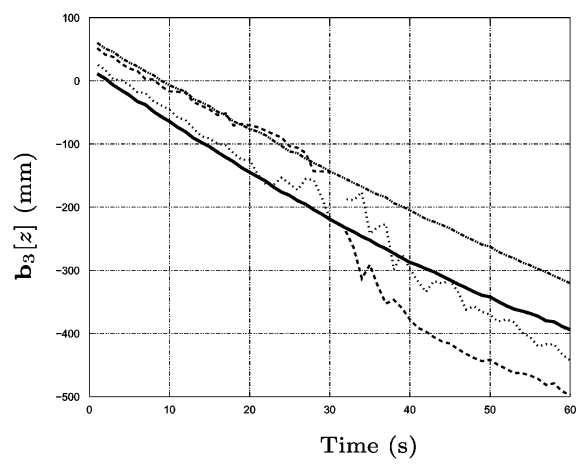

(c)

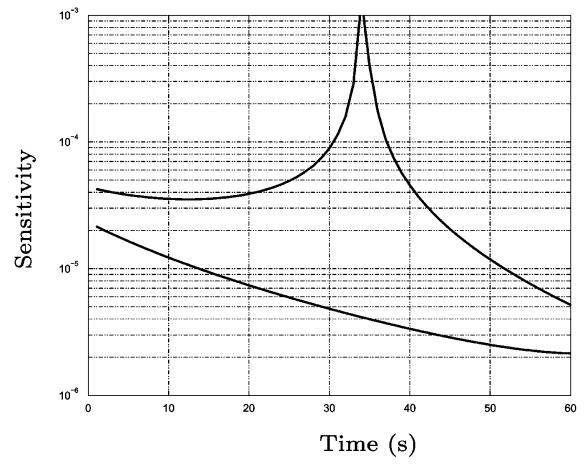

(f)

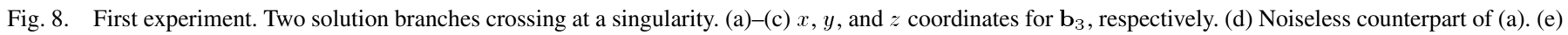
and (f) Sensitivity indices obtained from experimental data and noiseless simulated data, respectively.

plane. Since the result of the first trilateration must be taken in the right half-space, the number of forward kinematic solutions is reduced to four. Note that these four solutions always come in two couples sharing the same sensitivity index because the corresponding volumes of the tetrahedra involved in the second and third trilaterations are the same for them.

\section{A. First Experiment}

This experiment consists of approaching the robot end effector to the base plane by only rotating at constant velocity the second robot joint while it is kept parallel to the $x$ axis of the base frame drawn in Fig. 6(c). Fig. 7 shows how the six wire lengths evolve along this trajectory. Fig. 8(a)-(c) shows the coordinates of $\mathbf{b}_{3}$ compatible with these lengths obtained as the result of the three consecutive trilaterations, thus accumulating all possible errors. The solid lines correspond to the actual motion executed by $\mathbf{b}_{3}$. Fig. 8(d) shows the noiseless simulated counterpart of Fig. 8(a), which permits better understanding of the behavior of the different solution branches.

The interesting point about this experiment is that two of the four possible solution branches cross at the singularity located at $t=32$ (actually, a singularity can also be seen as a configuration in which two solution branches intersect). Note the great noise amplification near the singularity for these two crossing branches, while the other two remain stable, and how this noise results in no solution to the trilateration for certain points, leading to a discontinuity. This gives rise to an important question: when there is no solution for a trilateration operation due to errors, which is the best estimation for the solution? An easy way to generate an approximate solution is to argue that, because the negative value of the determinant inside the square

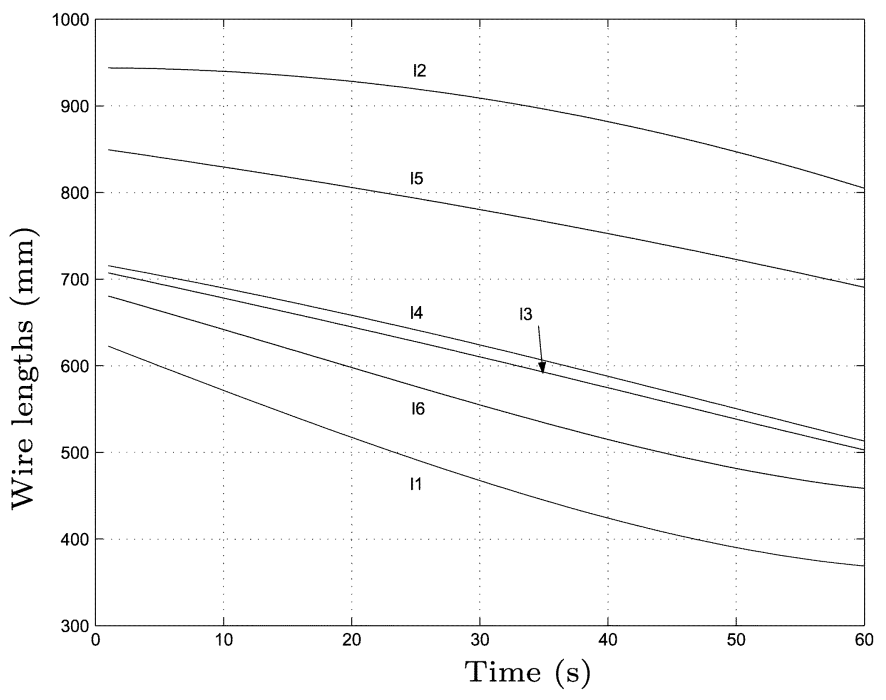

Fig. 9. Second experiment. Evolution of the six wire lengths along the trajectory.

root in the trilateration formulation outlined in Section II is due to errors in the lengths, it is reasonable to set this square root to zero. Unfortunately, this simple device does not always provide an acceptable approximation and, as shown in [11], one has to solve in general a nonlinear least square problem.

Fig. 8(e) and (f) shows the sensitivity index defined in (17) obtained from experimental and noiseless simulated data, respectively. Note that, while the two stable solutions keep a low sensitivity, that for the crossing solutions goes to infinity at the singularity. This means that the uncertainty ellipsoid in the configuration space of the platform becomes unbounded and at least 


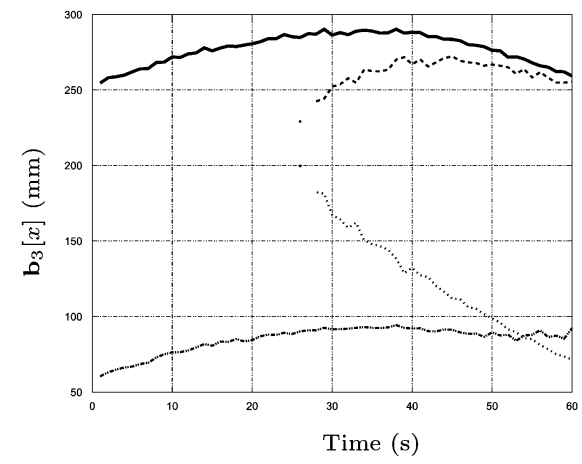

(a)

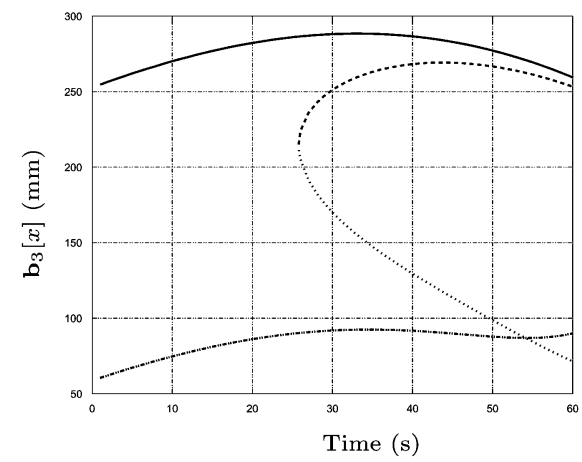

(d)

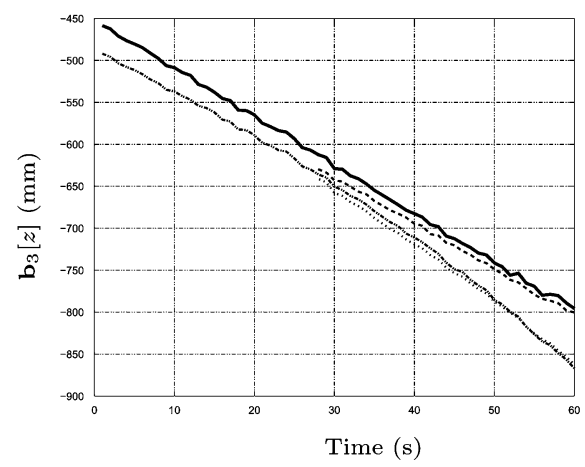

(b)

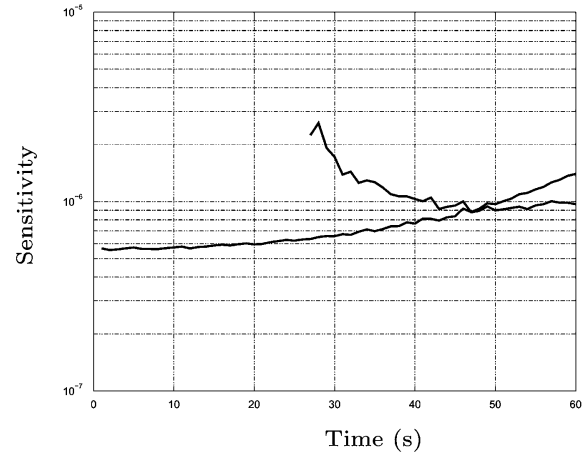

(e)

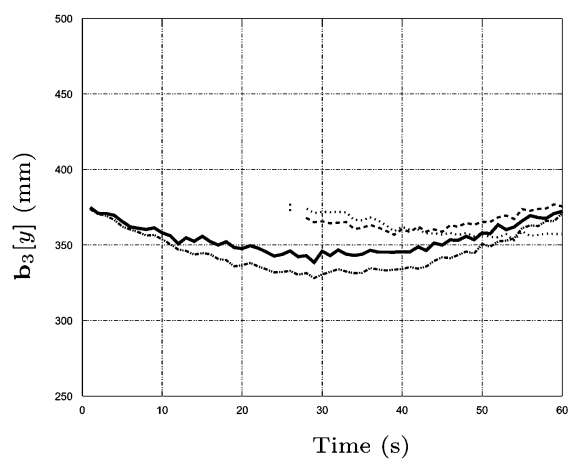

(c)

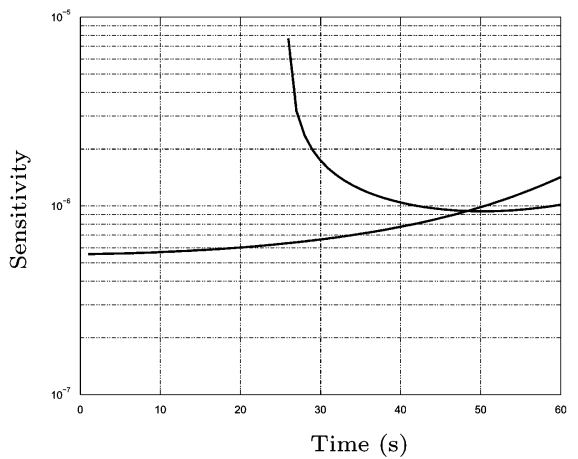

(f)

Fig. 10. Second experiment. Two solution branches vanishing at a singularity. (a)-(c) $x, y$, and $z$ coordinates for $\mathbf{b}_{3}$, respectively. (d) Noiseless counterpart of (a). (e) and (f) Sensitivity indices obtained from experimental data and noiseless simulated data, respectively.

one of the three tetrahedra involved in the trilaterations becomes planar. In this case, one of tetrahedra associated with the third trilateration degenerates.

At the beginning of a measuring cycle, the pose of the platform can be assumed to be known. This would require deciding the orientation of the tetrahedra involved in the second and third trilateration which could be done by visual inspection, or adding extra sensors such as inclinometers. The investigation on the optimal choice of the type and number of extra sensors to be used-in such a way a one-to-one correspondence between sensor measurements and the parallel manipulator pose is obtained-has been addressed from many points of view (see [25] and the references therein).

Without extra sensors, if a singularity is traversed, an ambiguity arises because two solution branches cross. In theory, since infinite accelerations are not physically feasible, it seems reasonable to design an algorithm able to track a unique solution based on the continuity of the velocity vector. Unfortunately, in practice, due to the noise amplification near a singularity, as this experiment has exemplified, this possibility seems unfeasible.

\section{B. Second Experiment}

This experiment consists of approaching the robot end effector to the base plane using a joint interpolated motion, that is, a straight line motion in the configuration space of the robot. Fig. 9 shows how the six wire lengths evolve along this trajectory. Fig. 10(a)-(c) shows the coordinates of $\mathbf{b}_{3}$ compatible with these lengths obtained as the result of the three consecutive trilaterations. The solid lines correspond to the actual motion executed by $\mathbf{b}_{3}$.
Fig. 10(d) shows the noiseless simulated counterpart of Fig. 10(a), which permits better understanding of the behavior of the different solution branches.

The interesting point about this experiment is that at the beginning of the trajectory only two solutions are possible. Actually, one of the tetrahedra associated with the third trilateration cannot be assembled with the lengths resulting from one of the two possible solutions for the second trilateration. At $t=27$, two other solutions arise to complete the set of four possible solutions. A closer analysis, based on noiseless simulated data [Fig. 10(d)], reveals that two solution branches come together in a singularity point with vertical tangent. As in the previous example, the exact point where the two solution branches meet cannot be exactly found experimentally because, due to error amplification near a singularity, no solution to some trilaterations can be found. Obviously, solution branches that come together and vanish at a singularity do not correspond to real solutions. Thus, although in theory up to four solutions are possible, two could be rejected on this basis.

Fig. 10(e) and (f) shows the sensitivity index obtained from experimental and noiseless simulated data, respectively. Note how the proposed sensitivity index is of practical interest as it provides a quality measurement of the estimations obtained directly from experimental data.

\section{Third Experiment}

There is no bias without noise. Then, the problem of exemplifying bias errors is that they are significant near singularities, precisely where noise is highly amplified, as has been shown in 


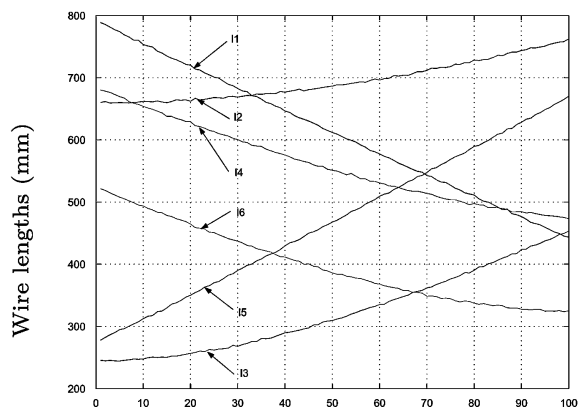

Time (s)

(a)

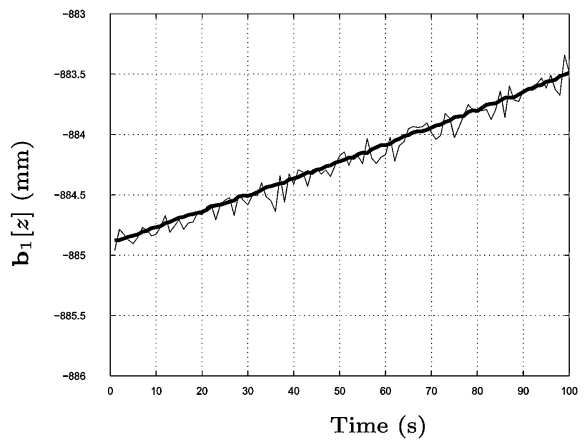

(d)

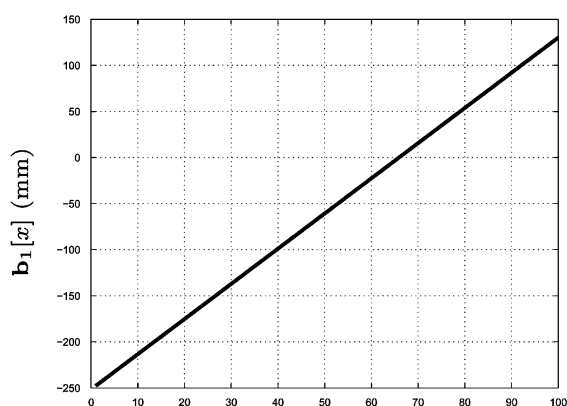

Time (s)

(b)

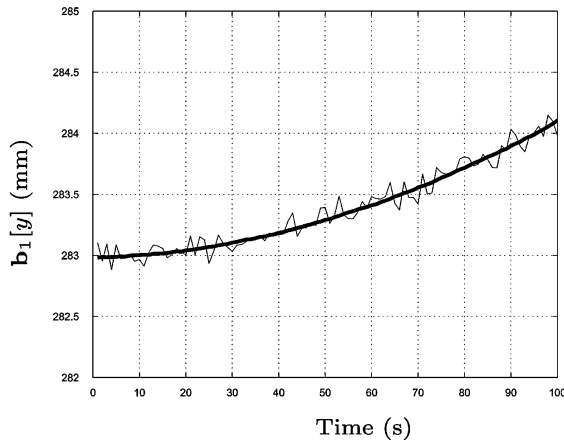

(e)

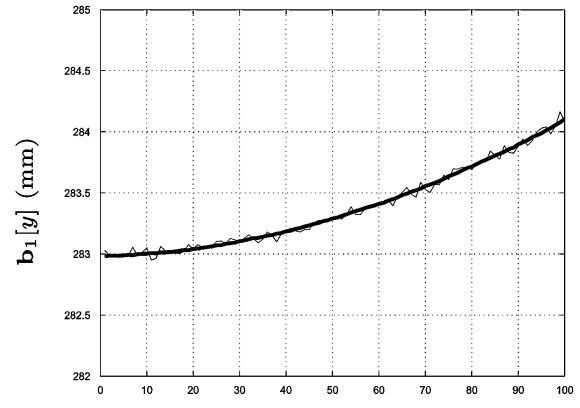

Time (s)

(c)

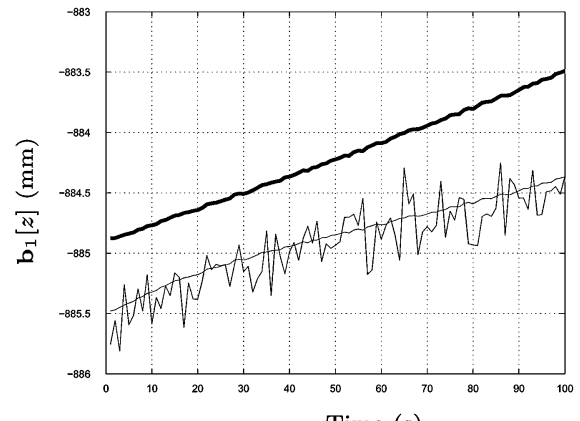

Time (s)

(f)

Fig. 11. Third experiment. The effect of bias errors. (a) Evolution of the six wire lengths. (b)-(d) $x, y$, and $z$ coordinates for $\mathbf{b}_{1}$, respectively. (e) and (f) $x$ and $y$ coordinates after adding Gaussian noise to the experimental data and averaging results.

the previous experiments. Since errors are assumed to have zero mean value, in this experiment we will try to distinguish bias from noise by averaging the results of multiple realizations of the same experiment.

This third experiment consists of moving the robot end effector so that the $y$ and $z$ coordinates of the platform attachments vary within a narrow range of $1 \mathrm{~mm}$ along a trajectory of $400 \mathrm{~mm}$. This permits expanding the scale for these two coordinates to analyze the effect of bias errors in detail. Fig. 11(a) shows how the six wire lengths evolve along the chosen trajectory. Fig. 11(b)-(d) shows the coordinates of $\mathbf{b}_{1}$ compatible with these lengths obtained as the result of the first trilateration.

The thick lines correspond to the actual motion executed by $\mathbf{b}_{1}$ obtained by simulation. The noisy plots correspond to the result of averaging 50 realizations of the same experiment. The results do not exhibit any apparent bias. This does not contradict the theoretical results obtained because the error in the wire lengths is quite low and the measurements have been taken next to the barycenter of the base points $\mathbf{a}_{1}, \mathbf{a}_{2}$, and $\mathbf{a}_{3}$ where the bias error is known to be minimum. Nevertheless, by adding Gaussian noise to the length measurements with a variance of $5 \mathrm{~mm}$ and averaging the results of 500 realizations, the $y$ coordinate is kept unbiased [Fig. 11(e)], but a clear bias error arises in the $z$ coordinate [Fig. 11(f)], that is, in the component orthogonal to the base plane. This fully agrees with the theoretical results outlined in Section V. Also, in Fig. 11(f), the bias error obtained from (24) with $\sigma_{r}=5 \mathrm{~mm}$ has been added to the result obtained without noise and plotted as a thin line. Since the final result is a good approximation of the averaged result with noise, it seems feasible to identify the variance of the wire-length errors by analyzing the bias errors.

\section{CONCLUSION}

A performance analysis of a 3-2-1 pose estimation device has been presented. It has been shown how, near a singularity, small errors in the wire lengths induce important errors in the pose estimations not only in terms of their variances but also in their biases.

Concerning the variances of the pose estimations, a sensitivity index for the estimations has been proposed which has proven to be of practical interest as it provides a quality measurement of the estimations obtained directly from experimental data.

Concerning the bias error, an asymptotic formula of remarkable simplicity for this error has been obtained thanks to a closed-form solution to the forward kinematics of the device in terms of Cayley-Menger determinants. It has been shown how all algebraic manipulations based on this formulation can be performed involving only distances, and the results can always been interpreted in terms of lengths, areas, and volumes. This has been revealed to be of great interest when calculating the required partial derivatives with respect to wire lengths to obtain the aforementioned asymptotic formula. It is also worth highlighting here that bias errors have been omitted in previous analysis of wire-based tracking devices.

Redundant measurements could be taken to reduce the effect of noise. However, a system of seven or more cables will invariably lead to entanglement problems, for which reason the presented technique is bound to encounter limitations in its scope.

The device has been analyzed for static pose estimations but, in general, this device has interest for estimating trajectories, that is, for tracking purposes. The measurements along a trajectory are not statistically uncorrelated so that they should be 
jointly smoothed during tracking to improve accuracy using, for example, a Kalman filter. The performance analysis given in this paper is of relevance to this end. For example, the characterization of the bias error must not be ignored at this point and it has to be suitably anticipated in this filter. This issue deserves further research.

\section{REFERENCES}

[1] Z. Geng and L. S. Haynes, "A 3-2-1 kinematic configuration of a Stewart platform and its application to six degree of freedom pose measurements," Robot. Computer-Integrated Manufact., vol. 11, no. 1, pp. 23-34, 1994.

[2] J. W. Jeong, S. H. Kim, and Y. K. Kwak, "Kinematics and workspace analysis of a parallel wire mechanism for measuring a robot pose," Mechanism Mach. Theory, vol. 34, no. 6, pp. 825-841, 1999.

[3] M. Griffis and J. Duffy, "A forward displacement analysis of a class of Stewart platforms," J. Robot. Syst., vol. 6, no. 6, pp. 703-720, 1989.

[4] P. Nanua, K. J. Waldron, and V. Murthy, "Direct kinematic solution of a Stewart platform," IEEE Trans. Robot. Autom., vol. 6, no. 4, pp. 438-444, Aug. 1990

[5] V. Parenti-Castelli and C. Innocenti, "Direct displacement analysis for some classes of spatial parallel machanisms," in Proc. 8th CISM-IFToMM Symp. Theory Practice Robots Manipulators, 1990, pp. $126-133$.

[6] P. Nanua and K. J. Waldron, "Direct kinematics solution of a special parallel robot structure," in Proc. 8th CISM-IFToMM Symp. Theory Practice Robots Manipulators, 1990, pp. 134-142.

[7] K. H. Hunt and E. J. F. Primrose, "Assembly configurations of some in-parallel-actuated manipulators," Mechanism Mach. Theory, vol. 28, no. 1, pp. 31-42, 1993

[8] D. Eberly, "Finding the intersection of three spheres," 1996, [Online]. Available: comp.graphics.algorithms newsgroup.

[9] H. Bruyninckx, "Forward kinematics for Hunt-Primrose parallel manipulators," Mechanism Mach. Theory, vol. 34, pp. 657-664, 1999.

[10] D. E. Manolakis, "Efficient solution and performance analysis of 3-D position estimation by trilateration," IEEE Trans. Aerosp. Electron. Syst., vol. 32, no. 4, pp. 1239-1248, Apr. 1996.

[11] I. D. Coope, "Reliable computation of the points of intersection of $n$ spheres in $\mathbb{R}^{n}$," Austral. New Zealand Ind. Appl. Math. J., pt. C, vol. 42, pp. 461-477, 2000.

[12] A. L. Mackay, "Generalized structural geometry," Acta. Crystalographica, vol. A-30, pp. 440-447, 1974.

[13] F. Thomas, E. Ottaviano, L. Ros, and M. Ceccarelli, "Coordinate-free formulation of a 3-2-1 wire-based tracking device using Cayley-Menger determinants," in Proc. IEEE Int. Conf. Robot. Automat., vol. I, 2003, pp. 255-363.

[14] L. M. Blumenthal, Theory and Applications of Distance Geometry. Oxford, U.K.: Oxford Univ. Press, 1953.

[15] A. Cayley, "A theorem in the geometry of position," Cambridge Math. J., vol. II, pp. 267-271, 1841.

[16] K. Menger, "New foundation for Euclidean geometry," Amer. J. Math., no. 53, pp. 721-745, 1931.

[17] O. Ma and J. Angeles, "Architecture singularities of platform manipulators," in Proc. 1991 IEEE Int. Conf. Robot. Automat., 1991, pp. $1542-1557$.

[18] J. M. McCarthy, Geometric Design of Linkages. New York: SpringerVerlag, 2000.

[19] D. M. Downing, A. E. Samuel, and K. H. Hunt, "Identification of the special configurations of the octaedral manipulator using the pure condition," Int. J. Robot. Res., vol. 21, no. 2, pp. 147-160, 2002.

[20] W. H. Press et al., Numerical Recipes in C, 2nd ed. Cambridge, U.K.: Cambridge Univ. Press, 1990.

[21] R. A. Johnson and D. W. Wichern, Applied Multivariate Statistical Analysis, 2nd ed. Englewood Cliffs, NJ: Prentice-Hall, 1988.

[22] E. Ottaviano, M. Ceccarelli, M. Totti, and C. Avila-Carrasco, "CATRASYS (Cassino tracking system): A wire system for experimental evaluation of robot workspace," J. Robot. Mechatron., vol. 14 , no. 1, pp. 78-87, 2002

[23] F. Thomas and L. Basañez, "Interactive planning using graphical simulation for robot task programming," in Proc. 10th CISM IFToMM Symp. Theory and Practice of Robots and Manipulators, 1993, pp. 378-385.

[24] M. Ceccarelli, E. Ottaviano, and M. Toti, "Experimental determination of robot workspace by means of CATRASYS (Cassino tracking system)," in Proc. 13th CISM-IFToMM Symp. Theory and Practice of Robots and Manipulators, 2000, pp. 85-92.
[25] V. Parenti-Castelli and R. Di Gregorio, "A new algorithm based on two extra-sensors for real-time computation of the actual configuration of the generalized Stewart-Gough manipulator," Trans. ASME, J. Mech. Design, vol. 122, pp. 294-298, 2000.

[26] F. Thomas and L. Ros, "Revisiting trilateration for robot localization," IEEE Trans. Robot., vol. 1, no. 1, pp. 93-101, Feb. 2005.

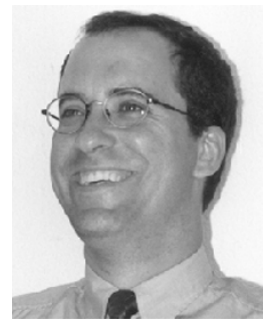

Federico Thomas received the B.Sc. degree in telecommunications engineering and the Ph.D degree (with honors) in computer science from the Technical University of Catalonia, Catalonia, Spain, in 1984 and 1988, respectively.

Since 1990, he has been a Research Scientist with the Industrial Robotics Institute of the Spanish High Council for Scientific Research, Barcelona, Spain. His research interests are in geometry and kinematics, with applications to robotics, computer graphics, and machine vision.

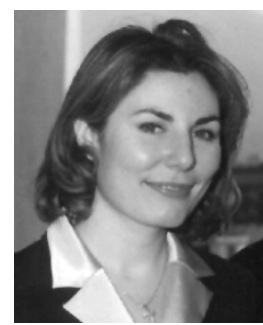

Erika Ottaviano received the degree (with honors) in mechanical engineering and the Ph.D. degree in applied mathematics from the University of Cassino, Cassino, Italy, 1998 and 2002, respectively.

In 2000, she was a Visiting Scholar with the Laboratory of Robotics, Department of Mechanical Engineering, Laval University, Quebec, QC, Canada. Since 2002, she has been an Assistant Professor of mechanics of machinery and mechanisms with the University of Cassino, where she teaches the course Mechanics of Automatic Machines and Mechanisms. Her research interests cover aspects of grippers and mechanics of robots, in particular workspace analysis and design of parallel mechanisms.

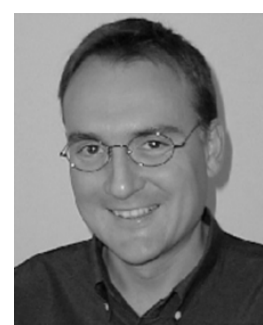

Lluís Ros received the mechanical engineering degree and the Ph.D. degree (with honors) in industrial engineering from the Technical University of Catalonia, Catalonia, Spain, in 1992 and 2000, respectively.

From 1993 to 1996, he was with the Control of Resources Group of the Cybernetics Institute, Barcelona, Spain, involved in the application of constraint logic programming to the control of electric and water networks. Since August 2000, he has been a Research Scientist with the Industrial Robotics Institute of the Spanish High Council for Scientific Research, Barcelona, Spain. His current research interests are in geometry and kinematics, with applications to robotics, computer graphics and machine vision.

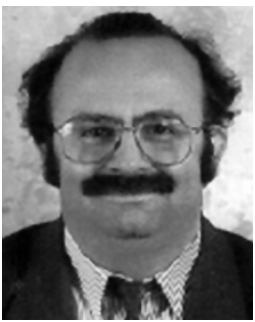

Marco Ceccarelli received the mechanical engineering degree (with honors) and the Ph.D degree in applied mechanics from the University "La Sapienza," Rome, Italy, in 1982 and 1988 respectively.

From 1987 to 1990, he was a Visiting Scholar and a Visiting Professor with Stanford University, Stanford, CA, and the Technical University of Valencia, Valencia, Spain, respectively. Since 1990 he has taught the courses Mechanics of Machinery and Mechanisms and Mechanics of Robots at the University of Cassino, Cassino, Italy. Since 1996, he has been the Director of the Laboratory of Robotics and Mechatronics of the Department of Mechanics, Structures, Environment and Territory, University of Cassino. In 2001, he was appointed a Full Professor of Mechanics of Machinery and Mechanisms at the same university. He authored the book Fundamentals of Mechanics of Robotic Manipulation (Norwell, MA: Kluwer, 2004).

Prof. Ceccarelli was Chairman of the Commission for History of Machine and Mechanism Science of the International Federation for the Promotion of Mechanism and Machine Science (IFToMM) from 1998 to 2004. He has been elected Secretary-General of IFToMM for the term 2004-2007. 